Table 1 Clinical characteristics

\begin{tabular}{lllc}
\hline Risk factor & LBBB $(\mathbf{n}=155)$ & STEMI $(\mathbf{n = 1 7 2 0})$ & $\mathbf{p}$ Value \\
\hline Mean age ( \pm SD) & $70.35 \pm 11.9$ & $64.95 \pm 14.0$ & $<0.0001$ \\
Male & $87(56.1 \%)$ & $1228(71.4 \%)$ & $<0.0001$ \\
Hypertension & $70(45.2 \%)$ & $668(38.8 \%)$ & 0.127 \\
Hypercholesterolaemia & $52(33.5 \%)$ & $512(29.8 \%)$ & 0.327 \\
Diabetes mellitus & $26(16.8 \%)$ & $201(11.7 \%)$ & 0.063 \\
Previous MI & $36(23.2 \%)$ & $205(11.9 \%)$ & $<0.0001$ \\
Previous CABG & $10(6.5 \%)$ & $44(2.6 \%)$ & 0.005 \\
\hline
\end{tabular}

Table 2 Clinical outcomes

\begin{tabular}{lllc}
\hline Outcome & LBBB $(\mathbf{n}=155)$ & STEMI $(\mathbf{n = 1 7 2 0})$ & $\mathbf{p ~ V a l u e ~}$ \\
\hline Door-to-balloon time $(\min \pm \mathrm{SD})$ & $40 \pm 17$ & $37 \pm 25$ & 0.710 \\
Call-to-balloon time $(\min \pm \mathrm{SD})$ & $128 \pm 36$ & $120 \pm 47$ & 0.263 \\
Acute coronary occlusion & $19(12.2 \%)$ & $1096(63 \%)$ & $<0.0001$ \\
PCl performed & $40(26 \%)$ & $1430(83 \%)$ & $<0.0001$ \\
30-day mortality (all) & $8(5.2 \%)$ & $120(6.9 \%)$ & 0.391 \\
30-day mortality (PCI) & $3 / 40(7.5 \%)$ & $94 / 1430(6.6 \%)$ & 0.825 \\
30-day mortality (no PCI) & $5 / 115(4.3 \%)$ & $26 / 290(8.9 \%)$ & 0.115 \\
Overall mortality & $32(27.8 \%)$ & $240(13.9 \%)$ & 0.023 \\
\hline
\end{tabular}

ST-elevation myocardial infarction (STEMI) referred to our tertiary centre for PPCI.

Methods All patients accepted for PPCI within the period of September 2009 to November 2011 were included in the study. Patient data obtained from our Cardiac Services Database System (Phillips CVIS) were analysed and angiographic images reviewed on our Cardiac Image Database (McKesson Horizon). Mortality data were gathered from the Summary Care Record (SCR) database. Statistical comparisons of continuous variables were made by an unpaired $t$ test. Categorical variables were compared using the $\chi^{2}$ test. A $p$ value of $<0.05$ was considered to indicate statistical significance.

Results During the study period, 1875 patients were referred for PPCI of whom 155 (8.3\%) had LBBB. Compared with STEMI, patients with $\mathrm{LBBB}$ were significantly older, more likely to be female and have prior history of MI and CABG (table 1). Patients with LBBB had similar door-to-balloon (DTB) and call-to-balloon (CTB) times. PCI was performed in 40 (26\%) patients with LBBB

\section{COMPARISON OF CLINICAL CHARACTERISTICS AND OUTCOMES IN PATIENTS WITH LEFT BUNDLE BRANCH BLOCK VERSUS ST ELEVATION MYOCARDIAL INFARCTION REFERRED FOR PRIMARY PCI}

N N Mannakkara, A M Mozid, R Showkathali, A S Sheikh, K H Tang, N M Robinson, A M Kabir, R 0 Jagathesan, J W Sayer, P A Kelly, R K Aggarwal, G J Clesham, J R Davies, R A Gamma The Essex Cardiothoracic Centre

\section{doi:10.1136/heartjnl-2013-304019.36}

Aims Current national and international guidelines continue to recommend activation of the primary percutaneous coronary intervention (PPCI) pathway in patients presenting with chest pain and presumed new-onset left bundle branch block (LBBB). Previous research has suggested that a lower proportion of patients presenting with $\mathrm{LBBB}$ require emergency intervention. In this study we have compared baseline clinical characteristics, angiographic findings and subsequent outcome in patients with LBBB versus

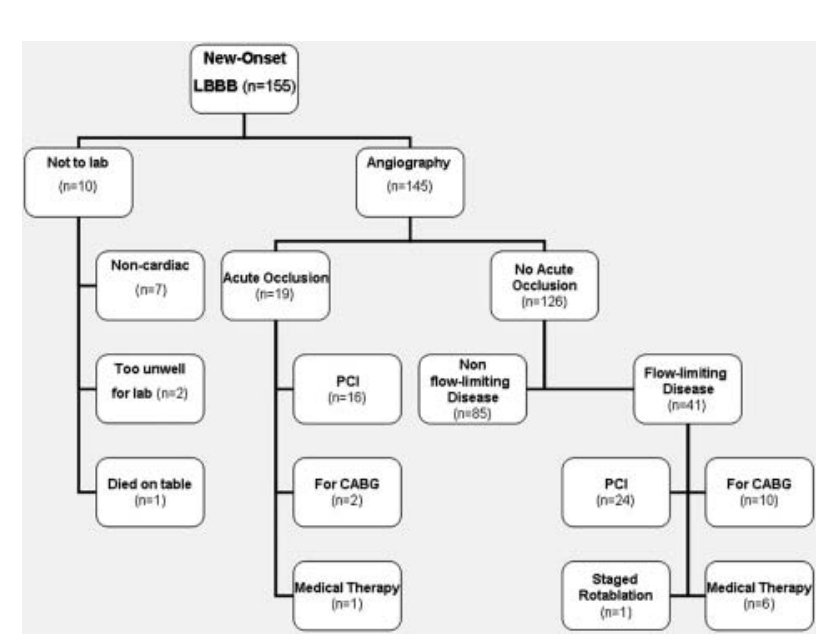

Figure 1 Clinical outcome in patients with LBBB. 


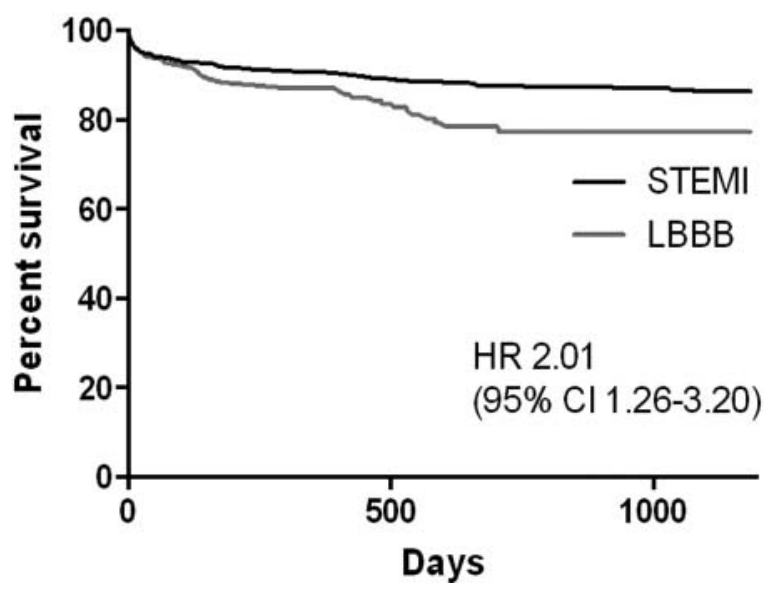

Figure 2 Kaplan-Meier comparison of survival curves.

although an acutely occluded culprit vessel was found in only 19 $(12.2 \%)$ patients (table 2). Furthermore, 85 (54.8\%) patients had non-flow limiting coronary artery disease and of those with significant disease $12(7.7 \%)$ patients required CABG (figure 1). Overall, an acute coronary syndrome (defined as ischaemic chest pain with positive troponin) was confirmed in only 67 (43.2\%) of patients presenting with LBBB. 30-day mortality was similar between LBBB and STEMI patients (table 2). However, during a mean follow-up period of 2.1 years, overall mortality was significantly higher in the LBBB group compared to STEMI (HR 2.01, 95\% CI 1.26 to 3.20 ) (figure 2).

Conclusions Our study shows that, in contrast to STEMI, only a small proportion of patients presenting with chest pain and LBBB had an acutely occluded coronary artery. Although short-term mortality was similar between the two groups, long-term outcome was significantly worse in patients with LBBB. Further work is needed to identify those patients presenting with $L B B B$ who are most likely to have an acute coronary occlusion, in order to facilitate the appropriate use of emergency coronary angiography and PPCI. 